\title{
Potential Impact of the Common Core Mathematics Standards on the American Curriculum
}

\author{
Hung-Hsi Wu
}

\author{
Department of Mathematics \#3840, \\ University of California, \\ Berkeley, CA 94720-3840. \\ E-mail: wu@berkeley.edu
}

In Y. Li, G. Lappan (eds.) (2014)

Mathematics Curriculum in School Education (pp. 119-142),

Advances in Mathematics Education, Dordrecht: Springer. 
Abstract

In June of 2010, the Common Core State Standards in Mathematics (CCSSM) were introduced in the U.S. Long before the advent of the CCSSM, American schools had a de facto national mathematics curriculum, namely, the curriculum dictated by school mathematics textbooks. While there are some formal differences among these books, the underlying mathematics is quite similar throughout. The resulting curriculum distorts mathematics in the sense that it often withholds precise definitions and logical reasoning, fails to point out interconnections between major topics such as whole numbers and fractions, and employs ambiguous language that ultimately leads to widespread nonlearning. The CCSSM make a conscientious attempt to address many of these problems and, in the process, raise the demand on teachers' content knowledge for a successful implementation of these standards. This article examines, strictly from an American perspective, some of the mathematical issues (primarily in grades 4-12) that arise during the transition from the de facto curriculum to the curriculum envisioned by the CCSSM. Although the CCSSM would seem to be strictly an American concern, these mathematical issues transcend national boundaries because there are very few deviations in the $\mathrm{K}-12$ curriculum across nations (for the $\mathrm{K}-8$ curriculum, see p. 3-31 to p. 3-33 of National Mathematics Advisory Panel, 2008).

Keywords: Common Core Standards, curriculum, content knowledge, definition, reasoning 


\section{Introduction}

In the unending search for improvement in mathematics education in the U.S. for the last half century, one thing seems to have been consistently overlooked; namely, the fact that there has been a de facto American mathematics school curriculum since the demise of the "New Math" in the early 1970s. This is the curriculum encoded in school textbooks. There are many textbooks, of course, and they are guided by quite different philosophical outlooks ranging from "traditional" to "reform". Nevertheless, the underlying mathematics is, overall, quite similar. While such a claim may startle some, the element of surprise will disappear the minute one considers for instance, the uniform lack of emphasis in school textbooks on giving precise definitions to concepts ${ }^{1}$ and, even more significantly, the same lack of emphasis on basing logical reasoning on precise definitions. If even this does not drive home the point, consider further the ambiguity of the meaning of fraction, multiplication or division of fractions, "variable", congruence, similarity, etc. How many textbooks explain how to multiply two fractions strictly on the basis of the definition of a fraction $?^{2}$ How many textbooks explain why any two circles are similar using a precise definition of similarity? And so on. This body of mathematical knowledge, contained in an overwhelming majority of school textbooks, will be henceforth referred to as Textbook School Mathematics (TSM). (See Wu, 20011a and 2011c, for a fuller discussion.) It will be seen from subsequent discussion that

\footnotetext{
${ }^{1}$ It should be understood that this article is primarily concerned with the mathematics in textbooks of grades 4-12. The need for correct and grade-appropriate definitions is no less acute in $\mathrm{K}-3$; for example, one does not want young children to be taught that a decimal is a number with a decimal point. Nevertheless, a short article such as this cannot adequately attend to all the instructional subtleties in those early grades.

${ }^{2}$ As an illustration of how definitions can be effectively used in mathematical reasoning even in a topic as elementary as fraction multiplication, one may consult Chapter 17 of $\mathrm{Wu}, 2011 \mathrm{~b}$.
} 
TSM, in the words of the Common Core State Standards for Mathematics $(2010),{ }^{3}$ page 3, "distorts mathematics and turns off students." More pertinent is the fact that much of the recent mathematics education crisis can be traced to the omnipresence of TSM in the school curriculum. The purpose of this article is to critically examine, strictly from an American perspective, several key areas of this de facto national curriculum from the vantage point of the CCSSM, highlight the deleterious effect of TSM, and give an indication of how the CCSSM - if they are faithfully implemented - might lead us out of the TSM jungle.

This de facto national curriculum has not been part of national dialog thus far for at least two reasons. The obvious one is the large grain size that is normally used in such general discussions. The other reason is very germane to this article: until recently, the issue of content in school mathematics education has not been on the frontline of this dialog. The failure to recognize this existence of the de facto national curriculum does carry serious consequences, however. In the writing of state or national mathematics standards, for example, the focus has always been on the optimal placement of standard mathematical topics in a certain grade band, e.g., addition of fractions in grades $4-6$, solving linear equations in middle school, triangle congruence criteria in high school, etc. The general expectation is that if the statement of the desired outcome (e.g., learn the addition of fractions and use it to solve problems) is phrased correctly, clearly, and in a grade-appropriate manner, and if it is faithfully implemented, progress will ensue (see, e.g., Carmichael et al., 2010, especially the Foreword). Such expectations ignore the

\footnotetext{
${ }^{3}$ Hereafter referred to simply as CCSSM.
} 
havoc that has been wrought by TSM in the school curriculum. Take, for example, the 2000 standards in grade 5 of California on the addition of fractions (p. 53 of Mathematics Framework for California Public Schools, 2006):

2.0 Students perform calculations and solve problems involving addition, subtraction, and simple multiplication and division of fractions and decimals:

2.3 Solve simple problems, including ones arising in concrete situations, involving the addition and subtraction of fractions and mixed numbers (like and unlike denominators of 20 or less), and express answers in the simplest form.

The statement of this standard is mathematically correct, ${ }^{4}$ and its placement in grade 5 is pedagogically unassailable, but now look what happens when it passes through the TSM melting pot and re-emerges in school textbooks:

1. Students are told to add fractions without being told precisely what adding fractions means, partly because there is no definition of a fraction as a number (this is universal practice).

2. Students learn the skill of adding fractions, either by drawing pictures but not given a formula (cf. Lappan et al., 1998a), or by being given a formula that uses the Least Common Denominator (Bennett et al., 2001, and Andrews et al., 2002). The reasoning is either not given or not given with focus and clarity.

\footnotetext{
${ }^{4}$ Although one may quibble with the restriction on the denominators used.
} 
3. When students are presented with a problem such as "How much water is in the bucket if you first pour in $2 \frac{3}{7}$ gallons and then another $3 \frac{2}{9}$ gallons", they dutifully use the method in step 2 only because "addition" is supposed to be used on account of the word "and", not because they know why.

There is a discussion in Wu (2011b), p. 221 and p. 228, about the correct definition of fraction addition and the reason why the Least Common Denominator should not be used to define the addition of fractions.

In any case, this is a glaring illustration of how good mathematical intentions are undermined by TSM-based implementations. There are countless examples of this, three major ones will be discussed at some length in a later section. The moral is that, until we eradicate TSM from the school curriculum, any mathematical standard that calls for the teaching of a mathematical topic in a certain grade will do nothing but rearrange the mathematically flawed presentations in TSM. Though not entirely appropriate, the proverbial "rearranging the deck chairs on the Titanic" does come to mind: it captures the zeitgeist of the situation.

The need to confront TSM in writing a set of standards was unimagined until the CCSSM came along. Anticipating the usual thinking of TSM, the CCSSM succeed, on the whole, in prescribing how each topic should be taught in a mathematically acceptable way. For example, here is how the CCSSM treat the addition of fractions: they ask that this skill be spread out through three grades. With drastic oversimplification, the CCSSM prescription goes something like this:

In Grade 3, understand a fraction as a number on the number line and 
interpret $\frac{m}{n}$ as $m$ copies of $\frac{1}{n}$; represent fractions on a number line diagram and explain equivalence of fractions in special cases, e.g., $\frac{1}{3}$ is the same point on the number line as $\frac{2 \times 1}{2 \times 3}$.

In Grade 4, explain why a fraction $\frac{a}{b}$ is equivalent to a fraction $\frac{n \times a}{n \times b}$ by observing that they are the same point on the number line. Also define addition of fractions as joining parts referring to the same whole. Then for two fractions with the same denominator, $\frac{m}{n}+\frac{k}{n}=\frac{m+k}{n}$.

In Grade 5, add and subtract fractions with unlike denominators by replacing given fractions with equivalent fractions, so that we have fractions with the same denominator. For example, $\frac{2}{3}+\frac{5}{4}=\frac{8}{12}+\frac{15}{12}=\frac{23}{12}$, which is joining copies of $\frac{1}{12}$ together.

Altogether, these standards guide students through three grades in order to help them understand the meaning of adding fractions. (For a more detailed presentation of how these standards can be implemented in the school classroom, one may consult pp. 9-13, 19-28, and 24-28 in Wu, 2011d.)

The end result is that addition is putting things together, even for fractions, and this mathematical development ends with the formula,

$$
\frac{a}{b}+\frac{c}{d}=\frac{a d+b c}{b d}
$$

with no mention of Least Common Denominator. What is obvious is that this presentation on adding fractions does not distort mathematics, and cannot be accused of turning students off because adding fractions is now seen to be no different from adding whole 
numbers: it's just putting things together.

In order to overcome TSM, the CCSSM have to be prescriptive, but the unprecedented prescriptive nature of CCSSM has provoked, not surprisingly, concerns about the possibility of stifling innovation and individualization (see, for example, page 6 of Institute for Research on Mathematics and Science Education, 2010). My interpretation of the situation is that, if all the innovations of the past decades could not produce a curriculum that does justice to mathematics, then it is time to try to prescribe a way out of this predicament. If we succeed in implementing the CCSSM and eliminating TSM in the process, then the time will come for a hundred flowers to bloom.

In a short article such as this, it is not possible to discuss TSM in detail, much less also discuss how the CCSSM try to counteract the ill effects of TSM. What I will do is to describe - in the broadest terms - some of the most salient features of TSM in the next section, and then discuss in greater detail three specific examples of how the CCSSM have responded to the challenge of TSM in the following section. The last section will contain a few comments about the potential impact of these proposed changes on teachers.

Now a word about citations of literature. To the extent that I am putting the whole system of school mathematics education under a microscope, any explicit citation in support of a particular statement is bound to give the false impression that I am targeting an author or a book. If I had a choice, I would rather not give any citations. However, the minimum requirement of scholarship dictates that I must, and the only way I can deal with this requirement is to enforce the policy of not citing any one source 
more than twice. Because this is a sensitive subject, I must add two more remarks in order to round off the picture. My citations were guided largely by what happened to be available to me at the time of writing, so that the presence or absence of a particular textbook or textbook series in the list of references has no significance beyond this fact. In addition, the quality of the cited textbooks varies, and it must not be assumed that each of them has all, or even most, of the flaws that are discussed in this article. I hope the reader will keep the last fact in mind.

\section{Overview}

The purpose of this section is to give a brief indication of some of the problems with TSM in the $\mathrm{K}-12$ curriculum.

The main topics of grades K-4 are place value and the whole number algorithms. In the de facto national curriculum, too often the standard algorithms are presented as faits accomplis that require neither motivation for their learning nor a clear explanation of why they provide the correct answers. More recently, these algorithms are downplayed in various ways: they are either buried in a host of other algorithms, or all the ingredients that lead to them are presented but the ultimate conclusions (the algorithms themselves) are not singled out, or they are de-emphasized in favor of invented algorithms (e.g., Bell et al. (2008) and Kliman et al. (2006)). Consequently, the fluent execution of the standard algorithms is also de-emphasized. What all these misguided approaches have in common is their failure to recognize the main mathematical message of these algorithms, which is to reduce all whole number computations to single-digit computations. The standard algorithms reduce a complicated task (the computation 
with multi-digit numbers) to a series of simple tasks (the computation with single-digit numbers) through the skillful use of place value. When the standard algorithms are taught from this perspective, they become a conduit to learning about two fundamental aspects of mathematics, namely, the need for logical reasoning and the fact that mathematics thrives on the reduction of the complex to the simple. See, for example, Chapter 3 of $\mathrm{Wu}, 2011 \mathrm{~b}$.

The CCSSM provide a remedy for the existing situation to a large extent. Concerning the multiplication algorithm, for example, they begin with a (too often neglected) definition of multiplication as repeated addition, e.g., $5 \times 7$ as $7+7+7+7+7$ (Standard 3.OA 1 in CCSSM, 2010), and then ask for the multiplication table to be committed to memory (Standard 3.OA 7 in CCSSM, 2010) in grade 3. The three basic laws of operation (commutative, associative, and distributive) are also introduced in grade 3. In grade 4, the CCSSM ask for the multiplication of "a whole number of up to four digits by a one-digit whole number," and the multiplication of "two two-digit numbers, using strategies based on place value and the properties of operations" (emphasis added; see Standard 4.NBT 5 in CCSSM, 2010). Finally in grade 5, students learn to multiply any two whole numbers. When the multiplication algorithm is taught in three grades as described, so that each step of this sophisticated algorithm is given ample time to be internalized by students, there is less of a chance that the teaching will be done by rote. This is all that one can ask for in a set of standards.

As mentioned earlier, the underpinning of these algorithms is place value, the fact that, for example, the 3 in 372 represents 300 and 7 is 70 while 2 is 2 . From a mathe- 
matical perspective, it may be more effective to explain to students, in a pedagogically appropriate way, the real reason that place value is needed: namely, to make it possible to count to any number, no matter how large, by limiting ourselves to the use of only ten symbols: $0,1,2, \ldots, 8,9$ (see Chapter 1 , Section 1.1 in $\mathrm{Wu}, 2011 \mathrm{~b}$ ). Thus place value is a property of the Hindu-Arabic numeral system we use and not a property of whole numbers. Exposing children to this fact at an early age would reinforce the importance of reasoning in mathematics. This is an idea that is worth exploring in the future.

The dominant topics of grades 4-6 (roughly) are fractions, decimals, and elementary geometry. There is no better illustration of the failure of the de facto national curriculum than the teaching of fractions. Fractions are students' first serious entry into abstractions. In their learning progressions, this is the first time that they can no longer rely on counting with their fingers (as they used to do with whole numbers) to relate what they are learning to their tactile experiences. They need detailed and careful guidanceincluding precise definitions of all the concepts as well as persuasive reasoning - in order to compensate for the loss of reliance on their fingers. Unfortunately, the response of the de facto national curriculum is to offer information that is at once confusing (e.g., a fraction is a part of a whole, a ratio, and a division) and misleading (the arithmetic operations on fractions bear no relation to those on whole numbers). In place of the precise definition of a fraction, it offers analogies, i.e., a fraction is like a piece of pizza or a shape in pattern blocks. In place of precise definitions for the arithmetic operations of fractions, it offers only algorithms and (of course) little explanation because it is impossible to explain anything that has not been precisely defined. The situation as 
described is so universal that no citation need be given: just open any school textbooks and this is all there is to see.

The same story is pretty much true of the teaching of decimals. Teaching decimals as an extension of whole numbers by the use of tenths, hundredths, etc.- but separate from fractions - is just another form of teaching-by-analogy. (Once again, this practice is so universal that no citation is necessary.) Indeed, this kind of teaching is only good for decimals with at most two decimal digits (pennies and dollars), so students do not get a precise conception of what a decimal is. In addition, such teaching is intellectually dishonest because, even for decimals with only three decimal digits such as 0.127 , the nomenclature of "one tenth and 2 hundredths and 7 thousandths" hides the fact that 0.127 is by definition a sum of fractions:

$$
0.127=\frac{1}{10}+\frac{2}{100}+\frac{7}{1000}
$$

Unfortunately, TSM has never been careful to teach decimals only after the addition of fractions has been defined. Historically, as well as conceptually, a decimal is a fraction whose denominator is a power of 10 . Once decimals have been integrated in this way into the domain of fractions, everything becomes simpler, be it the comparison of decimals or the computational algorithms with decimals, especially multiplication and division. (One can consult Sections 12.3, 13.4, 14.2, 15.3, 17.2, and 18.4 of Wu, 2011b.)

If I fault the de facto national curriculum for the flawed instruction on fractions, it is because the instruction is incommensurate with our expectations that students acquire a robust knowledge of fractions. If all we ask of students is that they achieve a passing acquaintance with the terminology of fractions, know roughly what they are, and be 
able to use them in simple everyday situations, then what TSM has to offer may just be good enough. Unfortunately, sophisticated word problems involving percent, ratio and rate await students in the sixth and seventh grades, and students need a thorough understanding of the division of fractions for their solutions, which in turn requires a solid foundation in the multiplication of fractions. The de facto national curriculum simply does not support this kind of learning. What we have is therefore a situation in which TSM teaches students only a little, but expects them to learn a lot. This sets students up to fail perfectly.

This fraction-decimal situation calls for change, and again the CCSSM have met this challenge to a large extent. Although fractions are introduced informally in grades 3 (as it should be), the recognition that a fraction is a point on the number line is encouraged from the beginning and the various basic theorems such as equivalent fractions are explained on this basis. Likewise, the arithmetic operations on fractions are defined and their algorithms explained in terms of the number line. The amount of details about the teaching of fractions that one finds in the CCSSM is unprecedented, and it raises the hope that a more sensible school curriculum on fractions will follow. As for decimals, the CCSSM state explicitly in grade 4, "Understand decimal notation for fractions, and compare decimal fractions." In other words, students are asked to learn that 0.127 is just a notation for the fraction $\frac{127}{1000}$. Thanks to the CCSSM, the teaching of decimals is now firmly integrated into the teaching of fractions. (For the details for both fractions and decimals, consult Wu, 2011b, Part 2.)

The other major topic of grades 4-6 is geometry, which is devoted mainly to the 
introduction of the basic vocabulary and the derivations of basic formulas pertaining to area and volume. The de facto national curriculum turns geometric instruction in these grades into a vocabulary-memorizing ritual, and not a very accurate one at that (see, for example, Andrews et al., 2002, or Bennett et al., 2001). For example, the statement in the CCSSM about "classifying two-dimensional figures in a hierarchy based on properties" (italics added; Standard 5.G 4) is a pointed reminder that the controversy about whether a square is a rectangle or whether a parallelogram is a trapezoid should be laid to rest. On the other hand, one of the most glaring omissions in the TSM presentation of area and volume formulas is the explanation of why the area of a rectangle with fractional side lengths is the product of the side lengths. This theorem, which is critical to the understanding of the concept of area as well as the concept of fraction multiplication in school mathematics (see pp. 62-64 of Wu, 2010a), seems to be missing in all existing textbooks and standards (e.g., Bell et al. (2008), National Council of Teachers of Mathematics ${ }^{5}$ (2000), and NCTM (2006)). It is to the credit of the CCSSM that they explicitly call for this explanation (standard 5.NF 4). Along this line, let it be mentioned that there is a common error in the proof of the area formula for a triangle:

$$
\text { area }=\frac{1}{2}(\text { base } \times \text { height })
$$

The argument given in textbooks, in an overwhelming majority of the cases, is only valid when the altitude meets the base, but not when the altitude falls outside the base, i.e., meets the line containing the base at a point outside the base (cf. Fuson (2006), and Kliman et al. (2006)). Unfortunately, if the area formula for a triangle is not known

\footnotetext{
${ }^{5}$ Hereafter referred to as NCTM.
} 
to hold in the latter case, i.e., when the altitude falls outside the base, it would be impossible to derive the area formula for a general trapezoid (see, for example, Wu, 2012, pp. 33-36 for the details). Corrections on this level are beyond the capability of a set of standards, even the CCSSM, but such curricular issues point to the overall logical oversight in TSM. An additional contribution of the CCSSM is their attempt to give at least an informal definition of area and volume. See Standard 5.NF 4 and Standard 5.MD 3 of CCSSM. Length and area are usually presented only as intuitive concepts in TSM in the elementary and middle grades, and this fact may be the cause of the well-known confusion concerning perimeter and area among students.

The emphases in grades 6-7 are on word problems involving percent, ratio, rate, and rational numbers. Before discussing these word problems, one must point out a grievous omission in the de facto national curriculum: the failure to make explicit the so-called Fundamental Assumption of School Mathematics (FASM), see Chapter 21 of Wu, 2011b. In essence, this is the statement that, although we only know how to compute with fractions (and later on, rational numbers) at this point, we can extrapolate formally the computational algorithms to all positive real numbers (respectively, all real numbers). FASM is conceptually important in the context of real-world problems about ratio and rate, and especially in algebra. The former often explicitly brings up numbers that are not necessarily fractions (e.g., the ratio of the circumference to the diameter of a circle). As to the latter, even the simplest identity such as

$$
\frac{1}{x-1}-\frac{1}{x+1}=\frac{2}{x^{2}-1}
$$

begs the question: what does this mean when (for example) $x=\pi$ if students are only 
taught the division of one rational number by another? (In this instance, one has to point out that FASM is not made explicit in CCSSM either.)

Problems involving percent, ratio, and rate are notorious for the amount of misunderstanding they elicit from students. The research on the probable cause of non-learning in ratio and rate has led to the emphasis on so-called proportional reasoning. As this will be discussed at some length in the example on Rate and Proportional Reasoning in the next section, we will merely mention the fact that, because these concepts have never been clearly explained (defined) in the de facto national curriculum, students cannot be in any position to provide solutions based on mathematical reasoning. Indeed, if there is no definition, there can be no valid reasoning. As the computer dictum goes: Garbage in, garbage out.

It must be pointed out that, although the CCSSM try valiantly to make some sense of this whole circle of ideas, they have not made any positive contributions in this direction. See the standards in $6 . \mathrm{RP}$ of grade 6 and $7 . \mathrm{RP}$ of grade 7 . On the other hand, the CCSSM have made great strides in elucidating another murky concept in the de facto national curriculum: the concept of an "expression". It would, however, be more appropriate to discuss this during the discussion of algebra below.

The teaching of rational numbers ${ }^{6}$ hinges on how negative numbers are integrated into students' knowledge of fractions. The de facto national curriculum relies mainly on manipulatives (e.g., the use of counters of different colors to represent positive and neg-

\footnotetext{
${ }^{6}$ In the education literature, the term rational numbers is generally taken to mean fractions. In mathematics, the term means positive and negative fractions. Because rational number is one of the most basic concepts in mathematics, it is best that people in education do not arbitrarily change accepted mathematical terminology.
} 
ative integers), analogies, and patterns (Usiskin et al., 1998, or Collins et al., 1998). The CCSSM acquit themselves particularly well in this regard by their insistence on the use of the number line and reasoning based on the general laws of operations (commutative, associative, and distributive laws) rather than patterns or manipulatives. See Standards 6.NS 5 and 6, and 7.NS 1 and 2. This is particularly true of the careful guided tour through the treacherous terrain of multiplication and division of rational numbers in Standard 7.NS 2. If so desired, one can consult Wu (2011c) for a leisurely discussion of teaching $(-a)(-b)=a b$ that is consistent with the CCSSM.

Grade 8 is a pivotal grade in the school mathematics curriculum, because it is in this grade that a decision is usually made as to whether the whole grade should be devoted to so-called Algebra I or simply make a beginning towards algebra. Now it must be said that there is no natural law that says students' learning of mathematics would suffer irrevocably if all the standard topics of Algebra I were not covered in grade 8. Moreover, what has been glossed over in any such discussion is the fact that the teaching of Algebra I in grade 8 according to TSM is accomplished at an unconscionable cost: it omits any mention of similar triangles, thereby cutting out the mathematical underpinning that connects the geometry of lines to the algebra of linear equations. Consequently, students are forced to learn by rote that one can get the slope of a line by choosing any two points on the line, and they are also forced to memorize by brute force the four forms of the equation of a line (often without success). We will examine further this issue in the example on Slope of $A$ Line in the next section.

In addition to the omission of any serious discussion of similar triangles, the middle 
school geometry curriculum according to TSM is a mélange of informal and disconnected discussions of diverse topics. Thus the concepts of translations, reflections, and rotations are taught as fun activities that heighten our sensibilities in art appreciation, e.g., Escher's prints. But are they relevant to mathematics? That is not so clear (see, e.g., Chapter 9 of Davison (2001) or Eicholz et al. (1995)). Congruence is just "same size and same shape", and its relationship with translations, reflections, and rotations may or may not be mentioned in passing (cf. Larson et al., 1999). Likewise, similarity means "same shape but not necessarily the same size", and no effort is made to show how this definition is related to the definition of similar triangles in terms of equal angles and proportional sides. In the rare event that such an attempt is made, it is not done in a mathematically disciplined way (cf. Lappan et al. (1998b)).

The above discussion points to two serious gaps in the de facto national curriculum: an explanation of why the graph of a linear equation of two variables is a line, and a smooth transition from middle school geometry to the high school geometry. Given the traditional curricular structure of the Algebra I-Geometry-Algebra II sequence in high school, the CCSSM had to solve the knotty problem-in the standards of grade 8 - of how to restructure the middle school geometry curriculum so that it provides a geometric foundation to fill both of these gaps. We now give a brief description of the restructuring (it is entirely consistent with the one given in $\mathrm{Wu} 2010 \mathrm{a}$ ).

The CCSSM accomplish this goal by asking for an intuitive exploration and discussion of translations, reflections, and rotations and for a definition of congruence as a finite composition of these rigid motions in the eighth grade (Standards 8.G 1-3). The CCSSM 
also call for an intuitive exploration and discussion of dilations, and the definition of a similarity transformation as the composition of a dilation and a congruence; then they call for an informal proof, in grade 8, that two triangles are similar if two pairs of angles are equal (Standards 8.G 4-5). The latter is the critical fact needed for the proof that the definition of the slope of a line is well-defined, and that the graph of a linear equation in two variables is a line (see, for example, $\mathrm{Wu}$ (2010b), Section 4). Then in high school, the definitions of translations, reflections, rotations, and dilations are formalized and congruence and similarity transformations are defined as in the eighth grade. These precise definitions can now serve to prove the usual criteria for triangle congruence (Standards G-CO 5 in high school geometry) and triangle similarity (Standards GSRT 2-3 in high school geometry). At this point, the usual development of Euclidean geometry may be pursued if so desired. In particular, translations, reflections, rotations, and dilations - basic concepts in advanced mathematics - are now fully integrated into school geometry as foundational concepts rather than as afterthoughts, and the proofs of theorems in plane geometry are now grounded in the tactile concepts of these basic transformations rather than in a set of abstract axioms. See Wu, 2010a and 2012.

In the context of teaching algebra, grade 8 is, of course, more than just the teaching of linear equations. This is also where other foundational algebraic concepts are developed and, among these, none is more basic than the proper use of symbols. It can be said that the de facto national curriculum really goes astray at this juncture: instead of making a smooth transition from arithmetic to algebra by carefully introducing the concept of generality and showing why the use of symbols is inevitable, this curriculum places 
the spurious mathematical concept of a "variable" front and center. On this shaky foundation, it introduces the concepts of algebraic expression, equation, and solving equations. This curricular development in TSM leads to misconceptions that make the learning of algebra unnecessarily difficult. To a very large extent, these misconceptions have been removed in the CCSSM. Specifically, the preamble to the high school algebra standards on p. 62 of CCSSM (2010) states:

An expression is a record of a computation with numbers, symbols that represent numbers, arithmetic operations, exponentiation, and, at more advanced levels, the operation of evaluating a function.

Back in grade 6 , standards 6 .EE 2c and 6.EE 6 already begin to clarify what a variable really is (i.e., a descriptive piece of terminology for a symbol) and what an expression is. Furthermore, standard 6.EE 5 clarifies what an equation is and standard A-REI (in high school algebra) explains what it means to solve an equation. These will be further discussed in the example on Solving Equations in the next section (for the details, see $\mathrm{Wu}, 2010 \mathrm{~b}$, Sections 1-3). Because of the ubiquity of equations and expressions in introductory algebra, these are genuine contributions to improving student learning.

We only have space to briefly mention the high school curriculum. It goes without saying that the de facto national curriculum has its usual share of flaws, e.g., lack of clarity and purpose in presenting the laws of exponents (e.g., Chapters 7 and 9 of Hoffer, Koss, et al. (1998), and Chapter 8 of Larson et al. (2007)), failure to define a parabola correctly (e.g., Chapter 8 of CME Project: Algebra 1 (2009), and Chapter 5 of Hoffer, Koss, et al. (1998)), failure to underscore the importance of completing the square in 
the study of quadratic functions (e.g., Chapter 5 of Holliday et al. (2008), or pp. 215220 and 491-504 in Murdock, Kamischke, and Kamischke (1998)), lack of clarity in presenting inverse functions and logarithms (e.g., Sections 7-2, 9-1, and 9-2 in Holliday et al. (2008), or Section 7.4 in Murdock, Kamischke, and Kamischke (1998)), etc. But let us address the global problems. In most schools, the traditional curriculum of Algebra I-Geometry-Algebra II is used, while some others follow the American integrated curriculum. ${ }^{7}$ While the artificial separation of the former into a full year of algebra or geometry is undesirable in principle, the latter has also been criticized for its imprecision, mathematical incoherence, and lack of mathematical closure, at least judging by what has been produced thus far (see e.g., Gray (undated) and Wu (2000)). The CCSSM chose to stay neutral on this issue by listing only what they call "conceptual categories" and leave the precise articulation of the high school curriculum to each state. This then leaves room for a third kind of curriculum that could possibly avoid both kinds of pitfalls, namely, one that is aligned with what is done in Japan (see, e.g., Kodaira (1992), (1996), (1997)) and other Asian countries in the Far East. To achieve this goal, one has to be aware of the need to structure mathematical topics in the CCSSM coherently. Moreover, one must be aware of the omissions of some standard topics in the conceptual categories of the CCSSM, e.g., the concept of the discriminant of quadratic polynomials, the explicit definitions of certain key concepts such as similarity and inverse functions, the fundamental algebraic properties of the exponential and logarithmic functions, etc.

\footnotetext{
${ }^{7}$ It is sometimes claimed that, because other nations adopt an integrated curriculum, so should we. This claim is misleading because the integrated curriculum of other nations is very different from the American integrated curriculum. The former is organized according to the internal development of mathematics whereas the latter seems to revolve around applications or "real world" problems.
} 
A full discussion of these issues would require a separate article.

\section{Some Examples}

The purpose of this section is to give a more detailed discussion of three key topics in school mathematics to illustrate the main difference between TSM and the curriculum envisioned by the CCSSM. In the first two of these (Solving Equations and Slope of A Line), the CCSSM excel, but in the third (Proportional Reasoning and Rate), the CCSSM do less well. I hope the choices I have made reflect my desire to give a balanced view of the CCSSM.

\section{Solving Equations}

What does it mean to solve an equation? To simplify the discussion, let us take a simple linear equation $4 x-3=2 x$. According to TSM, solving an equation requires a confrontation with a "variable". From a typical textbook, we have the following:

A variable is a letter used to represent one or more numbers. An algebraic expression consists of numbers, at least one variable, and operations. An equation is a mathematical sentence formed by placing the symbol "=" between two algebraic expressions. A solution of the equation is a number so that when it is substituted for the variable in the equation, the equality is true. (Collins et al. (1998), pp. 800-808.)

In this view of algebra, a variable is something distinct from numbers. Since all that students know up to this point are numbers (and geometry), a variable is a mysterious object. That said, here are the usual steps for solving $4 x-3=2 x$ : 
Step 1: $-2 x+(4 x-3)=-2 x+2 x$.

Step 2: $2 x-3=0$

Step 3: $(2 x-3)+3=0+3$

Step 4: $2 x=3$

Step 5: $x=\frac{3}{2}$

How do we justify Step 1 (adding $-2 x$ to both sides), for example, if we don't know what a variable is? Since a variable is a mystery, the equality $2 x-3=4 x$ is even more of a mystery. Adding the "variable" $-2 x$ to both sides deepens the mystery.

There seem to be three strategies in TSM to deal with this mysterious step of removing $2 x$ from both sides. First: Invoke the principle (first enunciated by Euclid) that equals added to equals remain equal (Larson et al. (2007), p. 154). This is comforting until one asks what is "equal"? If we don't know what either side means, how do we know they are "equal"? Second: Use algebra tiles to "model" this solution of $4 x-3=2 x$. Thus let a green rectangle model a variable and a red square model -1 . Then it seems "natural" that, if we remove two green tiles on the left (i.e., adding $-2 x$ ), we should also remove two green tiles on the right (Bellman et al. (2007), p. 133).

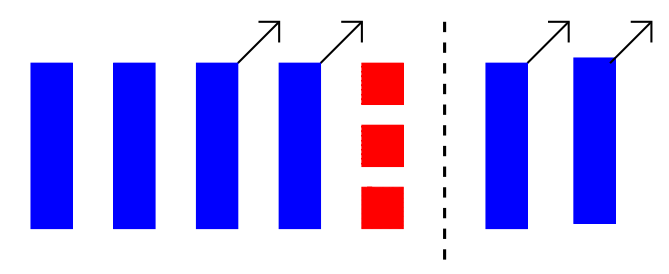

Third: Use a balance scale to "model" the equation $4 x-3=2 x$. It seems "obvious" that if we remove $2 x$ (whatever it is) from both weighing pans, the pans will stay in 
balance (Larson et al. (1999), p. 66).

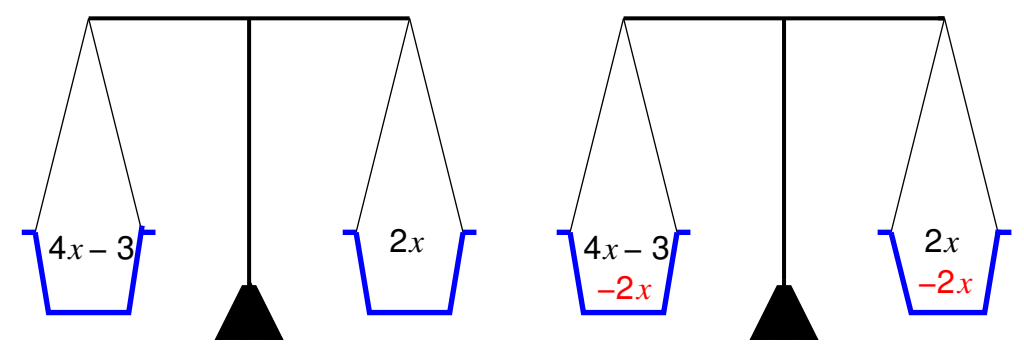

The other steps are justified in exactly the same way, making analogies using the intuitive meaning of "equality", algebra tiles, or balance scales.

These analogies are useful psychological ploys to win students' trust, but mathematics has to explain why something is true by logical reasoning, not by making sly suggestions about why it might be true because of analogies. By replacing reasoning with analogies, TSM guarantees that the fear of variables will live on.

The correct way to solve equations is well-known and very simple (cf. Wu (2010b), Section 3), but it took the CCSSM to finally incorporate it into a set of standards:

- (Grade 6, EE 5) Understand solving an equation or inequality as a process of answering a question: which values from a specified set, if any, make the equation or inequality true? Use substitution to determine whether a given number in a specified set makes an equation or inequality true.

- (High school Algebra, A-REI 1.) Explain each step in solving a simple equation as following from the equality of numbers asserted at the previous step, starting from the assumption that the original equation has a solution. Construct a viable argument to justify a solution method.

Let us see what it means to solve an equation from this perspective. The key idea is 
what may be called the Basic Protocol in the use of symbols: What a symbol stands for must be clearly stated when a symbol is introduced (Wu (2010b), p. 9). Armed with this idea, we can start anew. Let $x$ be a real number. An equation with $\boldsymbol{x}$, such as $4 x-3=2 x$, is a question asking whether the two numbers $4 x-3$ and $2 x$ are equal as numbers. It could be true, or it could be false. To solve the equation $4 \boldsymbol{x}-\mathbf{3}=\mathbf{2 x}$ is to determine all the numbers $x$ for which the equality is true.

We now show how to correctly solve $4 x-3=2 x$, but the principle holds in general (e.g., for polynomial equations). We first assume that there is a solution, i.e., there is a number $x_{o}$ so that $4 x_{o}-3=2 x_{o}$. Because we are now dealing with numbers, the previous five steps make perfect sense. Thus, starting with $4 x_{o}-3=2 x_{o}$, we get:

$$
\begin{aligned}
& \text { Step } i:-2 x_{o}+\left(4 x_{o}-3\right)=-2 x_{o}+2 x_{o} . \\
& \text { Step } i i: 2 x_{o}-3=0 \quad \text { (by use of the assoc. law for numbers) } \\
& \text { Step } i i i: \quad\left(2 x_{o}-3\right)+3=0+3 \\
& \text { Step } i v: 2 x_{o}=3 \quad \text { (by use of the assoc. law for numbers) } \\
& \text { Step } v: x_{o}=\frac{3}{2}
\end{aligned}
$$

Are we done? No. We have not proved that $\frac{3}{2}$ is a solution of $4 x-3=2 x$, only that if there is a solution, it must be equal to $\frac{3}{2}$. Having narrowed down the possible candidates to $\frac{3}{2}$, we can now complete the solution process by proving that $\frac{3}{2}$ is a solution with a simple computation:

$$
4\left(\frac{3}{2}\right)-3=2\left(\frac{3}{2}\right)
$$


because both sides are equal to 3 . This shows that the previous Steps 1-5 are a procedurally correct way to solve the equation. More importantly, this shows that Steps 1-5 actually make sense provided they are taught, not as computations with a mysterious quantity called a variable, but as computations with numbers. ${ }^{8}$

According to TSM:

Understanding the concept of variable is crucial to the study of algebra, and that a major problem in students' efforts to understand and do algebra results from their narrow interpretation of the term. (NCTM (1989), p. 102.)

On the contrary, a variable is not a mathematical concept. Imposing it on students as a mathematical concept can only obstruct their learning of algebra.

\section{Slope of A Line}

The concept of the slope of a line is a staple of grade 8 mathematics. In TSM, the definition of slope is the following: Let $L$ be a nonvertical line in the coordinate plane and let $P=\left(p_{1}, p_{2}\right)$ and $Q=\left(q_{1}, q_{2}\right)$ be distinct points on $L$. Then the slope of $L$ is defined to be $\frac{p_{2}-q_{2}}{p_{1}-q_{1}}$.

\footnotetext{
${ }^{8}$ Although our purpose is to expose the mathematical flaws of TSM, a side remark about the related pedagogical issue of how to implement the correct mathematics in the school classroom may not be out of place. In the context of solving equations, one may ask whether school students must solve equations in this formal and turgid fashion each time an equation is solved. The simple answer is no, because pedagogical common sense must be exercised. One suggestion is to explain in great detail-the first time an equation is solved - what the process described in Steps $i-v$ is all about. When the teacher feels comfortable that the students have understood the process, then they should be allowed to abbreviate their work more or less as in Steps $1-5$ on page 23 above.
} 
Is this well-defined, i.e., does it make sense?

Not yet, because if $A=\left(a_{1}, a_{2}\right)$ and $B=\left(b_{1}, b_{2}\right)$

are also on $L$, is the slope of $L$ equal to

$$
\frac{a_{2}-b_{2}}{a_{1}-b_{1}} ?
$$

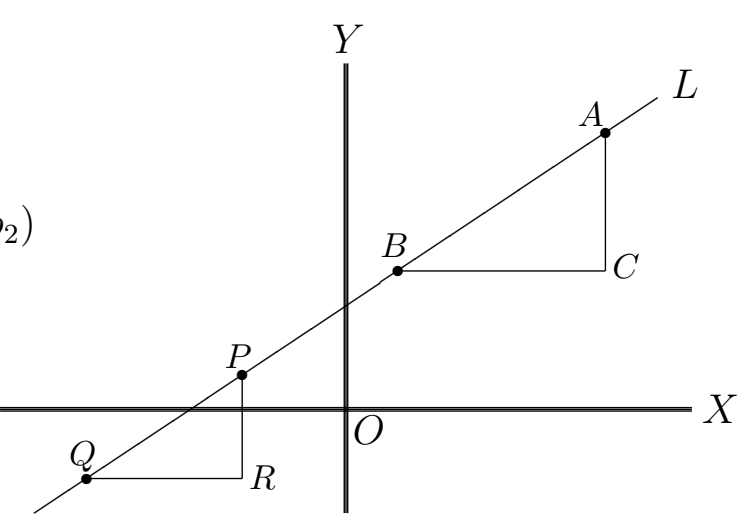

In other words, is it true that $\frac{p_{2}-q_{2}}{p_{1}-q_{1}}=\frac{a_{2}-b_{2}}{a_{1}-b_{1}} ?$

This question must be answered because slope is supposed to be a property of the line $L$ and not of the two points $P$ and $Q$ on $L$ that happen to be chosen. Students need the assurance that, if they happen to choose $A$ and $B$ on $L$, they still get the same number. Unfortunately, this question is not even raised in TSM, much less answered.

The proof of the equality $\frac{p_{2}-q_{2}}{p_{1}-q_{1}}=\frac{a_{2}-b_{2}}{a_{1}-b_{1}}$ requires the concept of similar triangles: $\triangle A B C \sim \triangle P Q R$. Assuming this similarity, then the proportionality of corresponding sides says

$$
\frac{P R}{A C}=\frac{Q R}{B C}
$$

Since the length of $P R$ is $p_{2}-q_{2}$, the length of $A C$ is $a_{2}-p_{2}$, etc., the equality is seen to be equivalent to the previous equality at least when the line $L$ is slanted to the right, as shown. If $L$ is slanted to the left, then the proportionality of the corresponding sides translates into the equality of the negative of the slopes, and the same conclusion prevails.

The TSM definition of slope confuses the slope of two chosen points on $\boldsymbol{L}$ with

\section{the slope of $L$.}

This kind of teaching-by-rote of slope has serious consequences in mathematics learn- 
ing. According to a recent survey of students' understanding of straight lines in algebra by Postelnicu and Greenes (2012), the most difficult problems for students are those requiring the identification of slope of a line from its graph. One can well imagine that if students do not realize they can use any two points on the line to compute its slope, they would be confused about "how to measure rise and run" (Postelnicu and Greenes, ibid.). The need for better teaching of the concept of slope is therefore real. Moreover, without a correct definition of slope, one cannot show that the graph of a linear equation $a x+b y=c$ is a (straight) line and the connection between the geometry of the line and the algebra of the equation will remain undeveloped. Students are therefore reduced to memorizing, by brute force, how to write down the equation of a line. Many are not successful.

The CCSSM prescribe a way out of this impasse. The standards in grade 8 ask that the following be done to make sense of "slope" as well as lay a foundation for high school geometry.

- Introduce rotation, reflection, translation (in the plane) and their compositions intuitively through hands-on activities, and define congruence as a composition of (a finite number of) rotations, reflections, and translations.

- Introduce dilation intuitively through hands-on activities, and define similarity as the composition of a dilation followed by a congruence.

- Give informal proofs of the basic criteria of triangle congruence.

- Give an informal proof of the $A A$ criterion of similar triangles: if two triangles 
have two pairs of equal angles, then they are similar.

- Use the AA criterion to show that the slope of a line is well-defined.

- Use the AA criterion to prove the Pythagorean Theorem.

See Chapters 4-6 in Wu 2010a, and the discussion of grade 8 in Wu 2012, for details.

The purpose of the emphasis on intuitive geometry is for students to gain the necessary geometric intuition as a preparation for the more rigorous course of high school geometry.

\section{Rate and Proportional Reasoning}

Proportional reasoning is supposed to be the "capstone of elementary school mathematics and the gateway to higher mathematics" (National Research Council (2001), p. 242). This term has come to mean "understanding the underlying relationships in a proportional situation" (p. 241 of National Research Council (2001)). Mathematics is about making explicit assumptions and then drawing logical conclusions from those assumptions. Unfortunately, what happens in TSM is that the "relationship in a proportional situation" is often hidden from students, thereby making it impossible for learning to take place. For example, consider the following prototypical problem in proportional reasoning:

A group of 8 people are going camping for three days and need to carry their own water. They read in a guide book that 12.5 liters are needed for a party of 5 persons for 1 day. How much water should they carry? (NCTM (1989), p. 83) 
Students cannot reason proportionally if they are not told that each person is assumed to drink roughly the same amount of water every day. Indeed they know from personal observations that different people drink different amount of water each day (at least before they get brainwashed by TSM), and therefore, without such an explicit assumption, they cannot possibly "think proportionally". Once this assumption is made explicit, however, students can experiment with, or can be shown, the numerical pattern in order to achieve some conceptual understanding of the situation. For example:

Let $\ell$ be the number of liters each person drinks each day, then two persons drink $\ell+\ell=2 \ell$ liters a day, three persons drink $\ell+\ell+\ell=3 \ell$ liters a day, $\ldots$, persons drink $\ell+\ell+\ell+\ell+\ell=5 \ell$ liters a day. Since we are given $5 \ell=12.5$, we have $\ell=2.5$ liters. Thus 8 people would need roughly $8 \times 2.5=20$ liters per day, so that they should carry $3 \times 20=60$ liters for three days.

This solution may not appear to be related to "relationships in a proportional situation", but, because it can be reformulated as follows, it is: For every positive integer $n$,

$$
\frac{\text { what } n \text { persons drink in } 1 \text { day }}{n}=\frac{n \ell}{n}=\ell
$$

Since the ratio $\ell$ is independent of $n$, we see that

$$
\frac{\text { what } 5 \text { persons drink in } 1 \text { day }}{5}=\frac{\text { what } 8 \text { persons drink in } 1 \text { day }}{8} \text {, }
$$

as both are equal to $\ell$. We now see explicitly the equality of two ratios. In particular, making use of the given fact that 5 persons drink 12.5 liters, we get

$$
\frac{12.5}{5}=\frac{\text { what } 8 \text { persons drink in } 1 \text { day }}{8} .
$$


Therefore, what 8 persons drink in one day $=(8 \times 12.5) / 5=20$ liters. In three days, 8 persons drink $3 \times 20=60$ liters, as before.

In retrospect, we see that the correctness of the following proportion,

$$
\frac{\text { what } m \text { persons drink in } 1 \text { day }}{m}=\frac{\text { what } n \text { persons drinks in } 1 \text { day }}{n} \text {, }
$$

for any positive integers $m$ and $n$ is a matter of logical reasoning once the needed assumption is revealed to students, but it is not any kind of a priori conceptual understanding that students can develop outside the mathematical framework. If we want students to learn to reason proportionally, then we should cleanse the curriculum of TSM and accord reasoning its rightful place.

A second kind of defect in the teaching of proportional reasoning is the inattention to precise definition. For example, here is another prototypical problem:

Which is the better buy: 12 tickets for $\$ 15.00$ or 20 tickets for $\$ 23.00$ ? (NCTM (2000), p. 221.)

Students need to be told, either in the problem itself or in general, the following two pieces of information: $(i)$ all tickets in each price group cost the same amount, and (ii) "better buy" means "the lower price per ticket." While neither is worth mentioning to an adult, an adolescent may well be learning his or her way in life at this point and therefore may not be aware of this information (or at least not the latter). In mathematics, one must strive for total clarity. Again, once these two facts are made explicit, students will see that,

if one ticket costs $d$ dollars, 2 tickets cost $d+d=2 d$ dollars, $\ldots$, and 12 
tickets cost $12 d$ dollars. Thus if $12 d=15$, then $d=1.25$ dollars. Similarly if another ticket costs $s$ dollars, 2 tickets cost $s+s=2 s$ dollars, $\ldots$, and 20 such tickets cost $20 s$ dollars. Thus if $20 s=23$, then $s=1.15$ dollars.

It follows that 20 tickets for $\$ 23.00$ is the better buy.

In both cases, TSM is guilty of withholding information and forcing students to make guesses. Mathematics is not about making the right kind of guesses, only about logical reasoning on the basis of an explicitly given hypothesis. It is also manifest that, once the proper information is supplied and students can see the reasoning behind such proportional reasoning problems, the solutions become entirely straightforward and therefore learnable. Let us therefore focus on removing these artificial obstacles imposed by TSM on learning.

The preceding problems are examples of a whole class of discrete problems on proportionality, in the sense that there is a "natural unit" to use in each problem (namely, one person or one ticket) and, furthermore, it is not necessary to go beyond this "natural unit" (there is no such thing as " 0.3 " person or $\frac{3}{4}$ ticket). For such discrete problems, the CCSSM do passably well by isolating the natural unit and the unit rate; see Standards 6.RP 1, 2, and 3a. However, there is another class of problems on proportionality, the so-called continuous problems where there is no natural unit; they are exemplified by constant speed, constant rate of water flow, constant rate of lawn mowing, etc. We can easily appreciate why there is no "natural unit" to measure time, for example: hour, minute, second, milli-second, micro-second, pico-second, etc., are all legitimate units to use for this purpose. These problems are special cases of what are known as "rate 
problems". For convenience, we will use rate to mean continuous rate in the rest of this article. ${ }^{9}$ We now turn our attention to these rate problems.

There are serious mathematical issues with the way rate problems are treated by TSM. The fact is that TSM conflates rate with constant rate. To understand this statement, we begin with a description of the underlying mathematics of the situation, one that requires calculus and is therefore not one that we can use with middle school students. For the sake of clarity, we will use speed exclusively in this discussion, but the idea is of course the same for other kinds of rate. Let $f(t)$ be the function that describes the distance of an object at time $t$, traveling along a (straight) line, from a fixed point $O$. Then the speed of the object at time $t$ is the derivative $f^{\prime}(t)$; the object is said to have constant speed $s$ if $s$ is a fixed number and $f^{\prime}(t)=s$ for all values of $t$. What is worth observing is that if the speed is not constant, the speed $f^{\prime}(t)$ varies with $t$ and there is no hope of expressing the speed ("rate') as the ratio of two numbers. On the other hand, if the speed is a constant $s$, then one can describe the "constant speed $s$ " for middle school students without resorting to calculus, as follows. Define the average speed over a time interval $[\boldsymbol{u}, \boldsymbol{v}]$ to be

$$
\frac{\text { difference in distance at time } u \text { and at time } v \text { from } O}{\text { length of the time duration from } u \text { to } v}=\frac{f(v)-f(u)}{v-u}
$$

Then an equivalent definition for the object to have constant speed $s$ is that its average speed over any time interval is equal to $s$. If we know that the speed is constant and is

\footnotetext{
${ }^{9}$ Note that we treat "rate" as a generic term that refers to a class of phenomena; each of the phenomena will have to be defined individually but there is a good reason not to try to define what "rate" means. Indeed, the general definition of "rate" as the derivative of the "work function" (a function of time) requires calculus; see the discussion of speed in the next paragraph. TSM makes believe that a term that requires calculus for its definition can nevertheless be bandied about in $\mathrm{K}-12$ as a precise concept. This is the reason why "rate" problem inspires such fear and loathing in schools.
} 
equal to $s$, then we can simply refer to $s$ as the speed of the object. In case of constant speed, then (and only then) is the rate (speed) the ratio of two quantities, as in the preceding equation.

With this understood, we can now gain a better understanding of how "rate" is mishandled by TSM. The following is a sample of some attempts to define "rate" by various textbooks; please take note that constant rate is implicitly assumed in each case.

A rate is a ratio that involves two different units. A rate is usually given as a quantity per unit such as miles per hour. This is called a unit rate. (Eicholz et al. (1995), p. 232.)

A quantity is a rate when its unit contains the word "per" or "for each" or some synonym. (Usiskin et al. (1998), p. 493.)

A rate can be thought of as an extended ratio, a ratio which enables us to think beyond the situation at hand, to imagine a whole range of situations in which two quantities are related in the same way. (Lamon (1999), p. 204.)

A rate is a comparison of the measures of two different things or quantities. The measuring unit is different for each value. (Van de Walle (1998), p. 293.)

In TSM, rate problems have to be done by assuming the constancy of rate, but because constant rate is never defined in TSM, no reasoning is possible in the solution of these problems. Instead, students are asked to memorize the following trinity of formulas,

$$
\begin{aligned}
\text { speed } & =\text { distance } / \text { time } \\
\text { time } & =\text { distance } / \text { speed } \\
\text { distance } & =\text { time } \times \text { speed }
\end{aligned}
$$


What is not commonly realized is that there is in fact no need to memorize anything in this situation, because the first is the definition of speed (when it is known to be constant), and the other two are consequences of the definition of division. Moreover, the resulting solution-by-rote is completely unnecessary because once "constant speed" is precisely defined, the solution can be obtained by mathematical reasoning. As illustration, consider the following problem:

John's grandpa enjoys knitting. He can knit a scarf 30 inches in 10 hours. He always knits for 2 hours each day.

1. How many inches can he knit in 1 hour?

2. How many days will it take Grandpa to knit a scarf 30 inches long?

3. How many inches long will the scarf be at the end of 2 days? Explain how you figured it out.

4. How many hours will it take Grandpa to knit a scarf 27 inches long? Explain your reasoning.

It is clear that, as is, the problem cannot be solved (except for part 2). Indeed, without knowing how much he knits in each of the ten 1-hour intervals, there is no way to answer part 1. Now, suppose we use the above definition of constant rate of knitting, and add the assumption that grandpa knits at a constant rate. Let us say he knits $\ell$ inches in a particular 1-hour interval, then the average rate of his knitting over this 1-hour interval would be $\frac{\ell}{1}=\ell$ inches per hour. But his average rate of knitting over a 10-hour interval, according to the given data, is $\frac{30}{10}=3$ inches per hour. By the assumption of constant 
rate, the two average rates are equal and therefore $\ell=3$ inches in that 1 -hour interval and, by assumption, in any 1-hour interval. The other parts can be solved similarly. We have therefore solved the problem by use of reasoning when the assumption of constant rate is added (perhaps this is what proportional reasoning means in TSM?).

Observe the commonality between the problem of knitting and the previous problem of 8 people camping: both become solvable only after the assumption of constant rate has been added.

To summarize, I hope I have explained clearly the flaws of "proportional reasoning" as it is understood in TSM. I wish I could say that the CCSSM are forceful and emphatic in exposing the need for a precise definition of constant rate as well as prescribing a remedy, but it must be said that while the CCSSM try to make sense of this circle of ideas, they have not made any serious headway. See Standards 6.RP $3 b$ and 3c, and Standards 7.RP 1 and 2. I should also add that there is no need for the CCSSM to be perfect in order to be worthy of support.

The previous remark concerning the need to add an assumption that all rates are constant rates might give the impression that the de facto national curriculum tries to make believe that every rate is constant in the real world. This is in fact not the case, because it does try to make students aware that even "speed" need not be constant. One cannot give a better illustration of this fact than to quote a 2011 test item for grade 8 in NAEP (National Assessment of Educational Progress (undated)):

3. For 2 minutes, Casey runs at a constant speed. Then she gradually increases her speed. Which of the following graphs could show how her speed 
changed over time?

A.

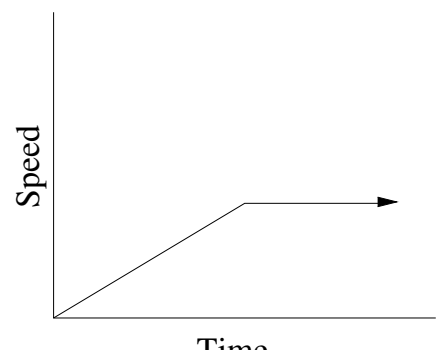

C.

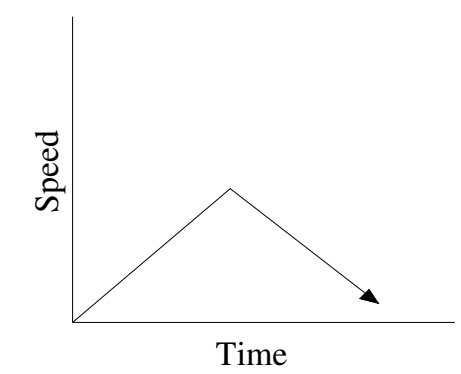

E.

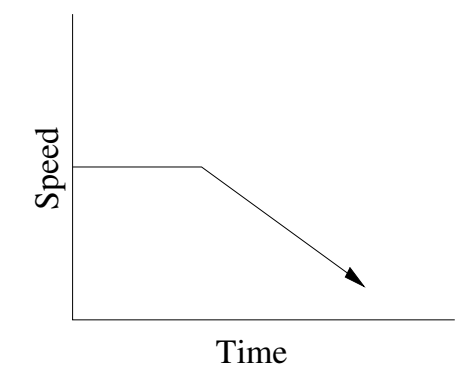

B.

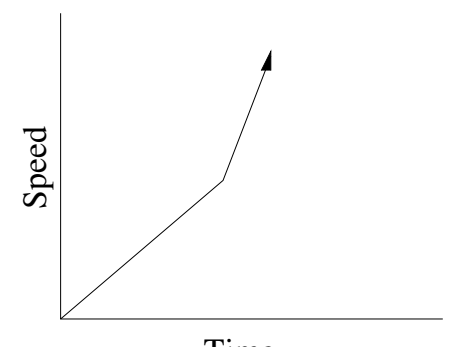

D.

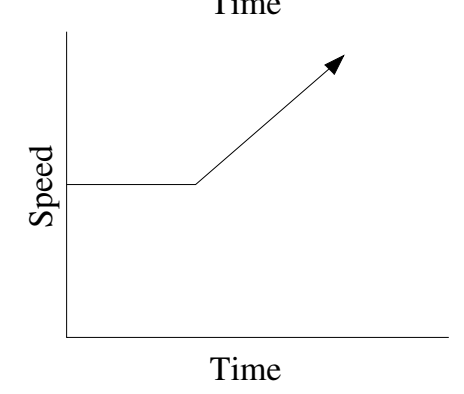

Here, the "speed" in the phrase "she gradually increases her speed" is clearly not one that is constant (regardless of the fact that the concept of variable speed cannot be defined in K-12). Thus TSM treats speed as automatically constant on the one hand, and wants students to be aware of non-constant speed on the other. Such blatant inconsistency is among the many reasons that TSM has to go.

\section{Concluding remarks}

To recap, we may describe the state of the American school mathematics curriculum as follows. For several decades, there has been a de facto national mathematics curriculum: the curriculum articulated in the school textbooks. The mathematics in these 
books is quite uniform in terms of its violation of the basic principles of mathematics (cf. the prefatory article To the Reader in Wu, 2011b), and we call it Textbook School Mathematics (TSM) to distinguish it from mathematics. For a long time, the school mathematics curriculum in each state has been drawn from TSM, so any significant curricular improvement will be difficult as long as TSM is recycled from generation to generation the way it is at present. Because commercial interests control textbook publishing, a direct attempt to change textbooks may be impossible without some outside stimulus. The CCSSM could be that needed stimulus. If they can spearhead a vigorous professional development program - nationwide - that allows our teachers to solidify their content knowledge, teachers will ultimately reject textbooks based on TSM. Then we can look forward to TSM's demise.

Such guarded optimism, however, is predicated on the assumption that the CCSSM are here to stay. The collapse of the "New Math" movement in the 1970's is a warning that unless the CCSSM can be implemented effectively in schools by knowledgeable teachers, the collapse of the CCSSM will also be inevitable. The survival of the CCSSM is therefore contingent upon our ability to produce a sufficiently large corps of mathematically knowledgeable teachers. To all who are dedicated to making good school mathematics education a reality, the inter-dependence of the survival of the CCSSM and the availability of knowledgeable teachers should spur us to fight for serious, contentbased professional development across the nation. We may add that the professional development must be one that can help teachers overcome their prolonged immersion in TSM. 
Thus far, there seems to be little awareness of the seriousness of the problem in both the education and mathematics communities, much less the will to bring about this kind of professional development. The difficulty of such an undertaking cannot be overstated. Given that our teachers are brought up on TSM in schools, and given that colleges and universities have done little to help preservice teachers realize that TSM is not mathematics, there is not likely to be significant change in the teacher pipeline anytime soon. As to teachers in the field, they are doubly betrayed: first by the sudden shift in our demand on their content knowledge, and then by our refusal to offer assistance. They are asked by the CCSSM to offer definitions to concepts that have never been properly defined for them: fractions, decimal, percent, expression, congruence, similarity... They are asked to offer explanations for skills that they were forced to learn by rote, such as invert and multiply, $(-a)(-b)=a b, \frac{-a}{b}=\frac{a}{-b}=-\frac{a}{b}$, writing the equation of a line, locating the minimum of a quadratic function... They are asked to teach certain facts as definitions and others as theorems, and they are uncertain which is which because TSM has never drawn a clear line between the two e.g., which of the following is a theorem and which is a definition?

$$
\begin{aligned}
& \frac{a}{b}=a \div b \\
& \frac{k}{\ell} \times \frac{m}{n}=\frac{k m}{\ell n}, \\
& a^{0}=1, \\
& 0 !=1,
\end{aligned}
$$

two lines are perpendicular if the product of their slopes is -1 , 
the graph of a quadratic function is a parabola.

They are also asked to look for structure in mathematics (p. 8 of CCSSM (2010)), but they have always been taught to consider whole numbers, fractions, and rational numbers as "different numbers" rather than as an orderly progression; length, area, and volume as distinctly different concepts rather than as special cases of geometric measurements; algebra as a separate subject from arithmetic rather than as a natural extension... They stand helpless, and our inaction keeps them helpless.

We can either wait for the inevitable collapse of the CCSSM, or we can firm up our resolve and confront the beast that is professional development. Which will it be?

Acknowledgement. I am grateful to Larry Francis for his corrections and useful suggestions. 


\section{References}

[1] Andrews, A. G. et al. (2002). Harcourt Math. Grade 5. Grade 6. (California Edition) Orlando, FL: Harcourt.

[2] Bell, M. et al. (2008). California Everyday mathematics. Teachers' Lesson Guide. Grade 4. Chicago, IL: McGraw Hill/Wright Group.

[3] Bellman, A. E. et al. (2007). Prentice Hall mathematics. Algebra 1. Boston, MA: Pearson/Prentice Hall.

[4] Bennett, J. et al. (2001). Scott Foresman California mathematics. Grade 4. Grade 5. Glenview, IL: Scott Foresman.

[5] Carmichael, S. B., Wilson, W. S., Porter-Magee, K., and Martino, G. (2010). The State of State Standards - and the Common Core-in 2010. Washington DC: Thomas B. Fordham Institute. Retrieved from http://www.edexcellence.net/ publications/the-state-of-state-of-standards-and-the-common-core-in-2010.html.

[6] CME Project: Algebra 1. (2009). Boston, MA: Pearson/Prentice Hall.

[7] Collins, W. et al.(1998). Glencoe Algebra I. New York, NY: Glencoe/McGraw-Hill.

[8] Common Core State Standards for Mathematics (2010). Retrieved from http://www.corestandards.org/the-standards/mathematics.

[9] Davison, D. M., Landau, M. S., McCracken, L., and Thompson, L. (2001). PreAlgebra. California Edition. Needham, MA: Prentice Hall. 
[10] Eicholz, R. E. et al. (1995). Addison-Wesley Mathematics. Grade 7. Grade 8. Menlo Park, CA: Addison Wesley.

[11] Fuson, K. C. (2006). Teacher's edition: Houghton-Mifflin math expressions. Grade 5. Boston, MA: Houghton-Mifflin.

[12] Gray, L. F. (undated). A sample list of errors in Core Plus materials. Retrieved from http://www.math.umn.edu/ ${ }^{\sim}$ gray/errors.html

[13] Hoffer, A. R., Koss, R., et al. (1998). Focus on Advanced Algebra. Menlo Park, CA: Addison Wesley.

[14] Holliday, B. et al. (2008). Glencoe McGraw-Hill Algebra 2. New York, NY: McGraw Hill.

[15] Institute for Research on Mathematics and Science Education (2010). Research in mathematics education: Where do we go from here? Retrieved from http://irmse.msu.edu/2010/11/16/washington-d-c-kick-off-event/irmseproceedings-mathematics-ed/.

[16] Kliman, M., Russell, S. J., Turney, C., and Murray, M. (2006). Investigations in number, data, and space in Grade 5: Building on numbers you know. Glenview, IL: Scott Foresman.

[17] Kodaira, K. (editor) (1992). Japanese grade 7 mathematics. Japanese grade 8 mathematics. Japanese grade 9 mathematics. Chicago, IL: University of Chicago. 
[18] Kodaira, K. (editor) (1996). Mathematics 1. Providence, RI: American Mathematical Society.

[19] Kodaira, K. (editor) (1997). Mathematics 2. Providence, RI: American Mathematical Society.

[20] Lamon, S. J. (1999). Teaching fractions and ratios for understanding. Mahwah, NJ: Lawrence Erlbaum.

[21] Lappan, G. et al. (1998a). Connected mathematics: Bits and pieces I and II. Menlo Park, CA: Dale Seymour.

[22] Lappan, G. et al. (1998b). Connected mathematics: Stretching and shrinking. Menlo Park, CA: Dale Seymour.

[23] Larson, R. et al. (1999). Passport to algebra and geometry. Evanston, IL: McDougall Littell.

[24] Larson, R. et al. (2007). McDougall Littell Algebra 1. Evanston, IL: McDougall Littell.

[25] Mathematics framework for California public schools (2006). Sacramento, CA: California Department of Education.

[26] Murdock, J., Kamischke, E., and Kamischke, E. (1998). Advanced Algebra Through Data Exploration. Berkeley, CA: Key Curriculum Press. 
[27] National Assessment of Educational Progress (undated). NAEP Questions Tool: Mathematics. Retrieved from http://nces.ed.gov/nationsreportcard/itmrlsx/search.aspx?subject=mathematics

[28] National Council of Teachers of Mathematics (1989). Curriculum and evaluation standards for school mathematics. Reston, VA: Author.

[29] National Council of Teachers of Mathematics (2000). Principles and standards for school mathematics. Reston, VA: Author.

[30] National Council of Teachers of Mathematics (2006). Curriculum focal points. Reston, VA: Author.

[31] National Mathematics Advisory Panel (2008). Foundations for Success: Reports of the Task Groups and Sub-Committees. U.S. Department of Education, Washington DC. http://www.ed.gov/about/bdscomm/list/mathpanel/reports.html

[32] National Research Council (2001). Adding it up. Washington, DC: National Academy Press.

[33] Postelnicu, V. and Greenes, C. (2012). Do teachers know what their students know? National Council of Supervisors of Mathematics Newsletter, 42 (3), 14-15.

[34] Usiskin et al. (1998). UCSMP Transition mathematics. Second edition. Glenview, IL: Scott Foresman Addison Wesley.

[35] Van de Walle, J. A. (1998). Elementary and middle school mathematics. New York, NY: Addison Wesley Longman. 
[36] Wu, H. (2000). Review of the Interactive Mathematics Program (IMP). Retrieved from http://math.berkeley.edu/ ${ }^{\sim}$ wu/IMP2.pdf

[37] Wu, H. (2010a). Pre-algebra. Retrieved from http://math.berkeley.edu/ ${ }^{\sim}$ wu/Pre-Algebra.pdf

[38] Wu, H. (2010b). Introduction to school algebra. Retrieved from http://math.berkeley.edu/ ${ }^{\sim}$ wu/Algebrasummary.pdf

[39] Wu, H. (2011a). Professional development and Textbook school mathematics. Retrieved from http://math.berkeley.edu/ ${ }^{\sim}$ wu/AMS_COE_2011.pdf.

[40] Wu, H. (2011b). Understanding numbers in elementary school mathematics. Providence, RI: American Mathematical Society.

[41] Wu, H. (2011c). Phoenix rising. Bringing the Common Core State Mathematics Standards to life. American Educator, 35(3), 3-13. Also: http://www.aft.org/pdfs/americaneducator/fall2011/Wu.pdf.

[42] Wu, H. (2011d). Teaching Fractions According to the Common Core Standards. Retrieved from http://math.berkeley.edu/ ${ }^{\sim}$ wu/CCSS-Fractions.pdf.

[43] Wu, H. (2012). Teaching Geometry According to the Common Core Standards. Retrieved from http://math.berkeley.edu/ ${ }^{\sim}$ wu/Progressions_Geometry.pdf. 